January 2006

\title{
Efficacy and safety of the six-dose regimen of artemether-lumefantrine in pediatrics with uncomplicated Plasmodium falciparum malaria: A pooled analysis of individual patient data
}

M. Makanga

Kenya Medical Research Institute

Zul Premji

Aga Khan University, zul.premji@aku.edu

C. Falade

University of Ibadan

J. Karbwang

World Health Organization

E.A. Mueller

Technical University

See next page for additional authors

Follow this and additional works at: http://ecommons.aku.edu/eastafrica_fhs_mc_pathol

Part of the Pathology Commons

\section{Recommended Citation}

Makanga, M., Premji, Z., Falade, C., Karbwang, J., Mueller, E., Andriano, K., Hunt, P., De Palacios, P. (2006). Efficacy and safety of the six-dose regimen of artemether-lumefantrine in pediatrics with uncomplicated Plasmodium falciparum malaria: A pooled analysis of individual patient data. American Journal of Tropical Medicine and Hygiene, 74(6), 991-998.

Available at: http://ecommons.aku.edu/eastafrica_fhs_mc_pathol/72 
Authors

M. Makanga, Zul Premji, C. Falade, J. Karbwang, E.A. Mueller, K. Andriano, P. Hunt, and P.I. De Palacios 


\title{
EFFICACY AND SAFETY OF THE SIX-DOSE REGIMEN OF ARTEMETHER-LUMEFANTRINE IN PEDIATRICS WITH UNCOMPLICATED PLASMODIUM FALCIPARUM MALARIA: A POOLED ANALYSIS OF INDIVIDUAL PATIENT DATA
}

\author{
MICHAEL MAKANGA, ZUL PREMJI, CATHERINE FALADE, JUNTRA KARBWANG, EDGAR A. MUELLER,* \\ KIM ANDRIANO, PHILIP HUNT, AND PATRICIA IBARRA DE PALACIOS \\ Kenya Medical Research Institute, Kilifi, Kenya/EDCTP, Tygerberg, South Africa; Muhimbili University College of Health Sciences, \\ Dar es Salaam, Tanzania; Department of Pharmacology and Therapeutics, College of Medicine, University of Ibadan, Ibadan, \\ Nigeria; Special Programme for Research and Training in Tropical Diseases, World Health Organization, Geneva, Switzerland; \\ Institute for Clinical Pharmacology, Public Health Research Association Saxony, Technical University, Dresden, Germany; Novartis \\ Pharmaceuticals Corporation, East Hanover, New Jersey; Novartis Horsham Research Centre, Horsham, United Kingdom; Novartis \\ Pharma AG, Basel, Switzerland
}

\begin{abstract}
Patient data from eight clinical trials were pooled and analyzed to study the efficacy and safety of the six-dose versus four-dose regimen of artemether-lumefantrine (coartemether; Coartem) in children weighing 5-25 kg. A total of 544 patients with uncomplicated $P$. falciparum malaria (six-dose: 343; four-dose: 201), matched for demographic and baseline characteristics and individual coartemether doses were included in the analysis. Analysis of day 28 cure rate based on the intention-to-treat and evaluable populations yielded corrected cure rates for the six-dose regimen of $93 \%$ and $96 \%$ compared with $61 \%$ and $76 \%$, respectively, for the four-dose regimen $(P<0.0001$ for both comparisons $)$. Similarly high cure rates were achieved with the six-dose regimen in non-immune infants weighing as little as $5 \mathrm{~kg}$. The six- and four-dose regimens were equally well tolerated. The main finding of this analysis is that the six-dose regimen of coartemether is safe and more efficacious than the four-dose regimen in children.
\end{abstract}

\section{INTRODUCTION}

There is a steady increase of drug-resistant $P$. falciparum malaria across many tropical areas. ${ }^{1}$ Malaria is estimated to cause about 1 million deaths/yr or 3,000 deaths/day, and most of these deaths occur in African children. ${ }^{2,3}$ In response to the anti-malarial drug resistance situation, the World Health Organization (WHO) recommends that treatment policies for falciparum malaria in all countries experiencing resistance to monotherapies should be combination therapies, preferably those containing an artemisinin derivative. ${ }^{4}$ It has been suggested that the widespread use of artemisinin-based combination therapies (ACT) could have a major impact on malaria. ${ }^{5}$ In fact, in areas of relatively low malaria transmission in Southeast Asia and South Africa, the widespread use of ACT has reduced the burden of malaria significantly. ${ }^{6}$ Although this benefit might be less marked in areas of very high transmission in Africa, it has been recently pointed out that ACT has the potential to be one of the greatest public health interventions for Africa this decade. ${ }^{7}$

Artemether-lumefantrine (coartemether; Coartem) (Novartis Pharma AG, Basel, Switzerland) is currently the only fixed-combination ACT widely available. Each tablet consists of $20 \mathrm{mg}$ artemether and $120 \mathrm{mg}$ lumefantrine. The rationale for this combination is that artemether rapidly reduces parasite biomass, and the long-acting lumefantrine eliminates residual parasites. Accumulating data suggest that, for optimum efficacy, coartemether should be given as six doses and with food containing fat. ${ }^{8}$ Although the four-dose regimen seemed sufficient to provide good cure rates in regions where patients were semi-immune and in areas without multidrug resis-

\footnotetext{
* Address correspondence to Edgar A. Mueller, Institute for Clinical Pharmacology, Public Health Research Association Saxony, Medical Faculty, Technical University, Fiedlerstrasse 27, D-01307 Dresden, Germany. E-mail: Edgar.Mueller@mailbox.tu-dresden.de
}

tance, ${ }^{9}$ WHO expressed the desire to have a single global dosing regimen for coartemether to avoid confusion and to assure its long-term effectiveness. However, the safety and tolerability of the higher dose regimen is an obvious concern in using the six-dose regimen as the global standard. Clinical studies investigating the six-dose regimen of coartemether showed high day 28 parasitological cure rates and good tolerability in adults and children from multi-drug-resistant areas of Southeast Asia and Africa. ${ }^{10-14}$ Moreover, a high adherence to the six-dose regimen was observed, with day 28 cure rates close to $100 \%$, irrespective of whether given under supervision or under unsupervised conditions of routine clinic practice in Africa. ${ }^{13,15}$ In a head-to-head study performed in Thailand, the six-dose administration schedule of coartemether was more efficacious and equally well tolerated than the four-dose regimen. ${ }^{10} \mathrm{~A}$ recently performed pooled analysis of data from randomized clinical trials confirmed the superior efficacy of the six-dose over the four-dose regimen in adolescents and adults, without altering tolerability and safety (E.A. Mueller and others, unpublished data).

Similar reassuring comparative data from children are lacking. In a multicenter non-comparative study in Kenya, Nigeria, and Tanzania, 310 children were treated with the six-dose regimen of coartemether. The drug was well tolerated, and the cure rate corrected by polymerase chain reaction (PCR) at day 28 was approximately $94 \% .{ }^{14}$ The study had no comparative four-dose regimen arm, for ethical reasons. It was considered that the four-dose regimen might not be sufficiently efficacious in nonimmune patients likely to be enrolled in that study. Against this background, we performed additional analyses on data assessed in children. Individual patient data from clinical trials performed under the auspices of coartemether's manufacturer in pediatric patients weighing between 5 and $25 \mathrm{~kg}$ were pooled to facilitate comparisons between the four-dose and six-dose regimens.

The aim of this pooled analysis was to confirm that the six-dose regimen of coartemether is more efficacious than 
the four-dose regimen in pediatric patients, while remaining equally safe and well tolerated. The results of the analysis were used for supporting the registration of the six-dose regimen as a single global dosing regimen, irrespective of age, body weight, immunologic status, and anti-malarial multidrug resistance.

\section{MATERIALS AND METHODS}

Trial identification and patient selection. Trials were considered eligible if they included children receiving the four- or six-dose regimen of coartemether for treatment of acute, uncomplicated $P$. falciparum malaria. All studies selected were performed under the auspices of the manufacturer of coartemether with similar endpoints, assessment schedule, and database structure. Eight studies were identified and provided individual patient data to be included in the pooled analysis (Table 1).

Some of the selected studies included adults and children or used a different dose regimen for certain weight groups (one half of the standard dose per administration). Also, the permitted range of baseline parasitemia varied between studies. These differences could have confounded comparisons between six-dose and four-dose regimens. To eliminate this confounding factor, only patients who met the following criteria were considered for pooling: 1) age $\leq 12$ years; 2) body

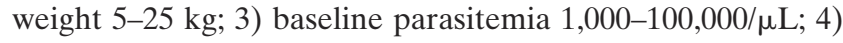
same individual dose of coartemether, according to body weight group allocation. These criteria were selected in a way to match the characteristics of the patients included in Study 2403 , which was the pivotal study to gain regulatory approval for using the six-dose regimen in kids. It was designed in partnership with the WHO.

All studies screened were conducted according to the Declaration of Helsinki with all its amendments. Informed consent was obtained from parents or legal guardians, with an appropriate institutional review board having approved the studies.

Study site, study design, and population. In the four-dose regimen group, there was a comparable distribution between African and Asian patients selected for pooling. Study 2403 was the primary source of data for the six-dose regimen and provided the vast majority of patients on this regimen. Studies 008, 025, and 026 included both adults and children. Although most studies were of open-label nature, the six-dose regimen group included a lower proportion of patients from doubleblind studies than the four-dose group (6\% versus 29\%). Comparators included sulfadoxine/pyrimethamine (SP, Study 010), chloroquine (Study 011), quinine (Study 003), and the combination of mefloquine and artesunate (MAS, Studies 008 and 026). In one case (Study 025), both coartemether regimens were compared within a study, in which two different six-dose regimens were used (same total dose), with doses given over 3 or 5 days; both six-dose regimens were considered for pooling. Studies 009 and 011 used a pediatric tablet formulation of coartemether (one half the dose of a standard tablet) that was produced during the original development program but is no longer available. To maintain comparability between dose regimens, only patients who received individual coartemether doses equivalent to the marketed formulation (i.e., two or four pediatric tablets per dose depending on body weight) were considered for pooling.

Before enrollment into the studies, male and female patients presenting with symptoms suggestive of malaria were assessed to be suffering from acute, uncomplicated $P$. falciparum malaria, and the diagnosis was confirmed by a positive blood smear. Patients with signs and symptoms of severe or complicated malaria were excluded.

In most of the trials, study endpoints included parasitological cure rate on days 7,14 , and 28 . Cure rates were not evaluated at day 28 in Studies 010 and 011. In Studies 003, 009, 025, 026, and 2403, PCR genotyping of paired blood samples was used to account for reinfections. Secondary outcomes included parasite clearance time (PCT), fever clearance time (FCT), and gametocyte carriage. Safety assessments consisted of monitoring and recording of all adverse events (AEs) and serious adverse events (SAEs), physical examinations, regular monitoring of hematology and blood chemistry, and regular recordings of electrocardiograms (ECGs) and vital signs. Two formulae (Fridericia and Bazett) were used to calculate the heart rate-adjusted QT interval (QTc).

Drug regimens. Table 2 summarizes the drug regimen of coartemether by body weight used in the trials that provided patients for pooling. Each standard tablet of coartemether contained $20 \mathrm{mg}$ of artemether and $120 \mathrm{mg}$ of lumefantrine, whereas the pediatric tablet formulation contained only 10 $\mathrm{mg}$ of artemether and $60 \mathrm{mg}$ of lumefantrine. For reasons outlined above, patients taking one or three pediatric tablets were excluded from pooling. Patients on the four-dose regimen received their doses at $0,6-8,24$, and 48 hours, whereas

TABLE 1

Summary of studies providing individual patient data for pooled analysis

\begin{tabular}{|c|c|c|c|c|c|c|}
\hline \multirow[b]{3}{*}{ Study no. } & \multirow[b]{3}{*}{ Region/year/study design } & \multirow[b]{3}{*}{ Enrolled patients } & \multicolumn{4}{|c|}{ Patients $(n)$ coartemether } \\
\hline & & & \multicolumn{2}{|c|}{ Overall* } & \multicolumn{2}{|c|}{ Qualified for pooling } \\
\hline & & & 4-dose & 6-dose & 4-dose & 6-dose \\
\hline$[009]^{16}$ & Gambia/1995-96/OL & 60 & 60 & 0 & 7 & 0 \\
\hline$[010]^{17}$ & Gambia/1996-97/DB,MC & 287 & 144 & 0 & 48 & 0 \\
\hline$[011]^{18}$ & Tanzania/1996/OL & 260 & 130 & 0 & 52 & 0 \\
\hline [003] & Thailand/1995-96/OL,MC & 219 & 79 & 0 & 55 & 0 \\
\hline$[008]^{19}$ & Thailand/1995-96/OL & 617 & 49 & 0 & 29 & 0 \\
\hline$[025]^{10}$ & Thailand/1996-97/DB,MC & 359 & 11 & 26 & 10 & 19 \\
\hline$[026]^{11}$ & Thailand/1997-98/OL,MC & 200 & 0 & 19 & 0 & 17 \\
\hline$[2403]^{14}$ & Kenya, Nigeria, Tanzania/2002-03/OL,MC & 310 & 0 & 310 & 0 & 307 \\
\hline Total & & 2312 & 473 & 355 & 201 & 343 \\
\hline
\end{tabular}

* All pediatric patients with 5- to $25-\mathrm{kg}$ body weight and treated with coartemether. DB, double-blind; MC, multicenter; OL, open-label. 
TABLE 2

Dose regimens of coartemether used by study

\begin{tabular}{|c|c|c|c|c|c|c|c|c|c|}
\hline \multirow[b]{2}{*}{ Weight group } & \multirow[b]{2}{*}{ Dose } & \multicolumn{8}{|c|}{ Study no. } \\
\hline & & 009 & 011 & 010 & 003 & 008 & $025^{*}$ & 026 & 2403 \\
\hline $5-10 \mathrm{~kg}$ & Four doses of one pediatric tablet & $\checkmark$ & $\checkmark$ & & & & & & \\
\hline $10-15 \mathrm{~kg}$ & Four doses of two pediatric tablets & $\checkmark$ & $\checkmark$ & & & & & & \\
\hline $15-20 \mathrm{~kg}$ & Four doses of three pediatric tablets & $\checkmark$ & $\checkmark$ & & & & & & \\
\hline $20-25 \mathrm{~kg}$ & Four doses of four pediatric tablets & $\checkmark$ & $\checkmark$ & & & & & & \\
\hline$<15 \mathrm{~kg}$ & Four doses of one standard tablet & & & $\checkmark$ & & & $\checkmark$ & & \\
\hline $15-25 \mathrm{~kg}$ & Four doses of two standard tablets & & & $\checkmark$ & & & $\checkmark$ & & \\
\hline 5 to $<10 \mathrm{~kg}$ & Six doses of one standard tablet & & & & & & & & $\checkmark$ \\
\hline 10 to $<15 \mathrm{~kg}$ & Six doses of one standard tablet & & & & & & & & $\checkmark$ \\
\hline 15 to $\leq 25 \mathrm{~kg}$ & Six doses of two standard tablets & & & & & & & & $\checkmark$ \\
\hline $10-20 \mathrm{~kg}$ & Four doses of one standard tablet & & & & $\checkmark$ & $\checkmark$ & & & \\
\hline $21-25 \mathrm{~kg}$ & Four doses of two standard tablets & & & & $\checkmark$ & $\checkmark$ & & & \\
\hline$<15 \mathrm{~kg}$ & Six doses of one standard tablet & & & & & & $\checkmark$ & $\checkmark$ & \\
\hline $15-25 \mathrm{~kg}$ & Six doses of two standard tablets & & & & & & $\checkmark$ & $\checkmark$ & \\
\hline
\end{tabular}

patients on the six-dose regimen received coartemether at 0 and 8 hours, and then twice daily on each of the following 2 days. In one treatment arm of Study 025, the six doses were administered over 5 days. Patients were advised to take coartemether with fat-containing food, if tolerated.

Data analysis. Efficacy parameters were defined as follows for the pooled analysis.

Day 7 cure rate. Proportion of patients with clearance of asexual parasitemia within 7 days of initiation of trial treatment, without subsequent recrudescence within these 7 days.

Day 14 cure rate. Proportion of patients with clearance of asexual parasitemia within 7 days of initiation of trial medication, without subsequent recrudescence within 14 days after study start.

Day 28 cure rate. The 28-day cure rate was considered to be of greatest importance, defined as the proportion of patients with clearance of asexual parasitemia within 7 days of initiation of trial drug, without subsequent recrudescence within 28 days after study start. Recrudescence was defined as the existence of positive blood smears after initial clearance of parasites from the peripheral blood. When available, results from PCR analysis were used to distinguish between recrudescence and reinfection.

Parasite clearance time. Time from first dose until first total and continued disappearance of asexual parasite forms that remained for at least a further 48 hours.

Fever clearance time. Time from first dose until the first time the body temperature decreased below and remained less than $37.5^{\circ} \mathrm{C}$ for at least a further 48 hours.

Gametocyte carriage. Proportions of patients with gametocytes at a given time point.

Subgroup analyses for efficacy were performed for patients 5 to $<10,10$ to $<15$, and 15 to $\leq 25 \mathrm{~kg}$. Safety was analyzed in terms of AEs, SAEs, clinical laboratory evaluations, and ECGs. AEs were defined as events that occurred after baseline but before recurrence of malaria.

Statistical analysis. All efficacy parameters were analyzed for the intent-to-treat (ITT) population, which was defined as all treated patients who met the pooling criteria and had confirmed malaria (i.e., baseline parasite count $\neq 0$ ). Of note, all patients who discontinued the study before evaluation of cure rate (day 7,14 , or 28 ) for any reason (including losses to follow-up) were considered a treatment failure in the ITT analysis for cure rates (i.e., conservative approach). Cure rates were also analyzed for the evaluable population (i.e., all patients in the ITT population who had parasite counts recorded up to the day of evaluation for cure rate: day 7,14 , or 28 ; if completed 7,14 , or 28 days) and took no other antimalarial drugs effective on $P$. falciparum or discontinued before day 7,14 , or 28 , as relevant, because of "unsatisfactory therapeutic effect" because of recurrence of $P$. falciparum. Safety was analyzed for the safety population, defined as all patients who received at least one dose of study medication.

For parasitological cure rates and gametocyte counts, a treatment comparison between the four- and six-dose regimen of coartemether was performed using the Fisher exact test, two-sided $(P \leq 0.05)$. Both, PCT and FCT were analyzed by survival analysis methods (Kaplan-Meier estimators with appropriate censoring and corresponding 95\% confidence intervals [CIs] for median event times). Otherwise, all data analyses were done using summary descriptive statistics.

\section{RESULTS}

Size of analysis populations. A total of 544 pediatric patients were included in the pooled analysis. Table 3 shows the numbers of patients in each defined analysis population. The 5 - to $<10-\mathrm{kg}$ body weight group treated with the four-dose regimen was small because of the exclusion from this population of patients who received doses of one pediatric tablet.

Patient disposition and baseline characteristics. The majority of patients completed the studies as planned, although the discontinuation rate was lower for the six-dose regimen than the four-dose regimen of coartemether (Table 4); the difference mainly was in the proportion of patients discontinuing because of unsatisfactory therapeutic effect $(0.6 \%$ versus $13.4 \%)$ and who were lost to follow-up (2.0\% versus $11.9 \%)$. The latter may have been related to better study monitoring and conduct in more recent studies (with the six-dose regimen) than in earlier trials (with the four-dose regimen). There was no difference in the rates of discontinuation caused by AEs, which were low for both regimens (six-dose: one patient $[0.3 \%]$; four-dose: two patients $[1.0 \%])$. One patient on the six-dose regimen died, which was unrelated to treat- 
TABLE 3

Number of patients on coartemether in each analysis population

\begin{tabular}{|c|c|c|c|c|c|c|c|c|}
\hline & \multicolumn{8}{|c|}{ Body weight range $(\mathrm{kg})$} \\
\hline & \multicolumn{4}{|c|}{ 4-dose regimen } & \multicolumn{4}{|c|}{ 6-dose regimen } \\
\hline & 5 to $<10$ & 10 to $<15$ & $15-25$ & Total & 5 to $<10$ & 10 to $<15$ & $15-25$ & Total \\
\hline Enrolled & 8 & 101 & 92 & 201 & 155 & 114 & 74 & 343 \\
\hline \multicolumn{9}{|l|}{ ITT population for } \\
\hline 7-day cure rate & 0 & 57 & 2 & 59 & 154 & 108 & 45 & 307 \\
\hline 14-day cure rate & 8 & 96 & 58 & 162 & 154 & 108 & 45 & 307 \\
\hline 28-day cure rate & 0 & 70 & 83 & 153 & 155 & 114 & 74 & 343 \\
\hline \multicolumn{9}{|c|}{ Evaluable population for } \\
\hline 7-day cure rate & 0 & 51 & 2 & 53 & 152 & 106 & 44 & 302 \\
\hline 14-day cure rate & 6 & 76 & 45 & 127 & 151 & 105 & 43 & 299 \\
\hline 28-day cure rate & 0 & 60 & 61 & 121 & 149 & 111 & 69 & 329 \\
\hline Safety population & 8 & 101 & 92 & 201 & 155 & 114 & 74 & 343 \\
\hline
\end{tabular}

Six-dose regimen: studies 025,026 , and 2403 .

ment (severe gastroenteritis contracted at home 5 days after the end of study treatment).

Overall, demographic and malaria-related characteristics at baseline were similar for the two coartemether groups (Table 4). Patients treated with the six-dose regimen tended to be younger and of lower body weight than those treated with the four-dose regimen. This reflected two factors: first, the exclusion of most patients in the $5-$ to $<10-\mathrm{kg}$ body weight group for the four-dose regimen because they received doses of one pediatric tablet, and second, there was an emphasis in Study 2403 on recruitment of patients in the lowest body weight group. Moreover, mean and median parasite density tended to be somewhat lower in patients who received the six-dose regimen than in those on the four-dose regimen.

Parasitological cure rates. Cure rates (Table 5) with the six-dose regimen were consistently higher than the four-dose regimen in all analysis populations, with the difference being greatest at 28 days. PCR-corrected day 28 cure rates were as high as $96 \%$ for the six-dose regimen in the evaluable popu-

TABLE 4

Patient disposition and baseline characteristics (safety population)

\begin{tabular}{|c|c|c|}
\hline & \multicolumn{2}{|c|}{ Coartemether } \\
\hline & 4-dose regimen & 6-dose regimen \\
\hline \multicolumn{3}{|l|}{ Patients, $n(\%)$} \\
\hline Enrolled & $201(100)$ & $343(100)$ \\
\hline Discontinued & $61(30.3)$ & $14(4.1)$ \\
\hline \multicolumn{3}{|l|}{ Sex, $n(\%)$} \\
\hline Male & $116(57.7)$ & $181(52.8)$ \\
\hline Female & $85(42.3)$ & $162(47.2)$ \\
\hline \multicolumn{3}{|l|}{ Age (years) } \\
\hline Median & 4.0 & 2.3 \\
\hline Range & $1-12$ & $0.2-12$ \\
\hline \multicolumn{3}{|l|}{ Weight (kg) } \\
\hline Median & 14.0 & 10.5 \\
\hline Range & $8-25$ & $5-25$ \\
\hline \multicolumn{3}{|c|}{ Body temperature $\left({ }^{\circ} \mathrm{C}\right)$} \\
\hline Median & 38.4 & 38.5 \\
\hline Range & $35.9-40.7$ & $36.7-40.9$ \\
\hline \multicolumn{3}{|c|}{ Parasite density $(\mu \mathrm{L})$} \\
\hline Median & 36250 & 17051 \\
\hline Mean \pm SD & $41,006 \pm 28,576$ & $30,930 \pm 31,700$ \\
\hline Range & $1,030-100,000$ & $1,000-100,000$ \\
\hline
\end{tabular}

Six-dose regimen: studies 025,026 , and 2403 . lation. In both the ITT and evaluable populations, the overall difference between the two coartemether treatments in terms of day 28 cure rates (with and without PCR correction) was clinically and statistically significant $(P<0.0001)$.

If analyzed by body weight, the six-dose regimen was again associated with consistently higher cure rates than the fourdose regimen. Body weight had little if any effect on cure rates with the six-dose regimen. PCR-corrected 28-day rates (evaluable population) for the 5 to $<10,10$ to $<15$, and $15-25$ $\mathrm{kg}$ body weight ranges were $97.3 \%, 94.6 \%$, and $97.1 \%$, respectively, for the six-dose regimen compared with $80.0 \%$ (10 to $<15 \mathrm{~kg}$ ) and $72.1 \%(15-25 \mathrm{~kg})$ for the four-dose regimen. There were too few patients treated with the four-dose regimen in the 5 - to $<10-\mathrm{kg}$ body weight group to allow meaningful cure rates to be calculated.

Times to parasite and fever clearance. Median PCT was shorter with the six-dose (24.2 hours; 95\% CI: $24.0,35.8)$ than the four-dose regimen (45.0 hours; $95 \%$ CI: 40.0, 48.0). The proportion of patients with rapid parasite clearance (i.e., within 48 hours) was more than $80 \%$ for the two regimens (six-dose: $94.2 \%$; four-dose: $83.1 \%$ ). Subanalyses according to body weight yielded similar results (data not shown).

Analysis of FCT data suggested a more rapid resolution of fever with the six-dose coartemether treatment regimen, al-

TABLE 5

Parasitological cure rates for coartemether by regimen

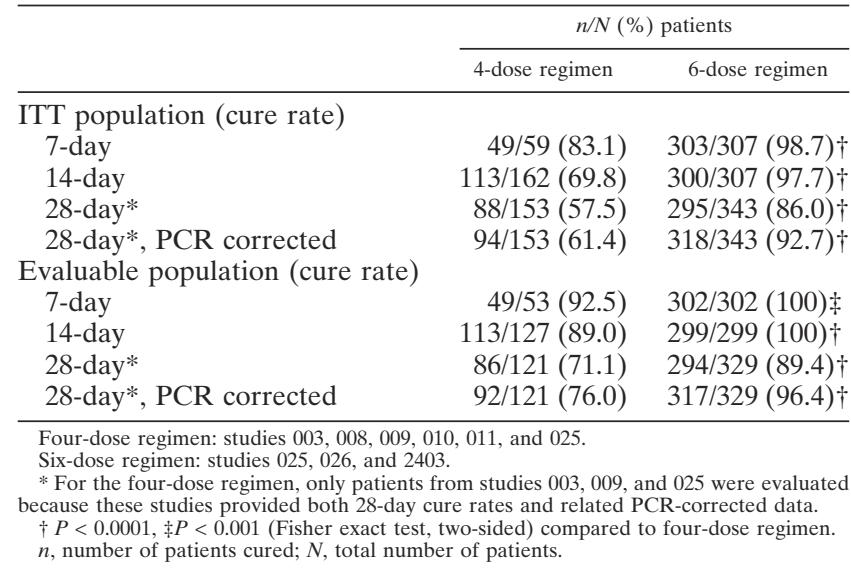


though results could have been confounded by the extensive use of antipyretic medications. For the six-dose regimen, median FCT was 7.9 hours $(95 \%$ CI: $7.8,7.9)$ and for the fourdose regimen was 24.0 hours (95\% CI: 23.8, 26.5). Again, subanalyses according to body weight yielded similar results (data not shown).

Gametocyte counts. The proportion of patients with gametocytes at early time-points (up to day 7) seemed to be lower for the six-dose regimen than the four-dose regimen, suggesting a rapid clearance of gametocytes, particularly with the six-dose regimen, or clearance of merozoites before gametocyte formation can occur (Table 6). Significantly fewer patients had circulating gametocytes at day 28 with the six-dose than the four-dose regimen. Subanalyses according to body weight were in line with the results obtained with the total population (data not shown).

Adverse events. Only three (0.9\%) and one (0.5\%) patient reported non-fatal SAEs in the six- and four-dose regimen, respectively. Of the SAEs occurring in patients on the sixdose regimen (convulsion, urticaria, viral hepatitis), only urticaria was suspected to be drug-related by the investigator. This case was classified as an SAE because the patient was hospitalized. The SAE reported in the four-dose group (pneumonia) was not considered to be drug-related.

The majority of patients reported at least one AE after baseline (Table 7). In both groups, most AEs were reported during the first 3 days of treatment, which were generally mild or moderate in severity. Severe AEs were infrequent (sixdose: $5.2 \%$; four-dose: $7.0 \%$ ). The most frequent AEs observed with both regimens included cough, anemia, anorexia, vomiting, hepato-/splenomegaly, headache, and diarrhea, most of which could have been disease related. ${ }^{20}$ The proportion of patients with AEs was similar between the 5 to $<10$, 10 to $<15$, and $15-25 \mathrm{~kg}$ body weight groups, with incidence rates of $75.5 \%, 72.8 \%$, and $77.0 \%$, respectively, for the sixdose regimen, compared with $87.5 \%, 91.1 \%$, and $91.3 \%$, respectively, for the four-dose regimen. There were some differences between body weight groups in particular AEs, such as headache or dizziness, which appeared less common in very small infants. This may have been related to the limited ability of these patients to communicate subjective symptoms without outward sign. Moreover, because the AEs accounting for the differences between body weight groups were generally of mild to moderate severity, those differences were not considered to be clinically relevant.

There were some notable differences between regimens in

TABLE 6

Proportion of patients with gametocytes by time-point (ITT population)

\begin{tabular}{lcc}
\hline & \multicolumn{2}{c}{ Patients $(\%)$} \\
\cline { 2 - 4 } & \multicolumn{3}{c}{ coartemether } \\
\cline { 2 - 3 } Day & $\begin{array}{c}\text { 4-dose regimen } \\
(N=201)\end{array}$ & $\begin{array}{c}\text { 6-dose regimen } \\
(N=343)\end{array}$ \\
\hline $0-3$ & 19.4 & $9.9^{*}$ \\
$4-7$ & 5.5 & $0.9^{*}$ \\
$8-14$ & 0 & 0 \\
$15-28$ & 3.0 & $0.3 \dagger$ \\
\hline
\end{tabular}

Four-dose regimen: studies 003, 008, 009, 010, 011, and 025 .

Six-dose regimen: studies 025,026 , and 2403 .

$* P<0.01, \uparrow P<0.05$ (Fisher exact test, two-sided) compared to four dose regimen $N$, total number of patients.
TABLE 7

Adverse events (after baseline but before recurrence of malaria) in more than $10 \%$ of patients receiving coartemether, irrespective of cause (safety population)

\begin{tabular}{lrr}
\hline & \multicolumn{2}{c}{$n(\%)$ patients } \\
\cline { 2 - 3 } & \multicolumn{2}{c}{ coaretmether } \\
\cline { 2 - 3 } \multicolumn{1}{c}{ Adverse event } & 4-dose regimen & 6-dose regimen \\
$(N=201)$ & $257(74.9)$ \\
\hline Total & $183(91.0)$ & $77(22.4)$ \\
Cough & $37(18.4)$ & $77(22.4)$ \\
Anemia & $49(24.4)$ & $63(18.4)$ \\
Anorexia & $80(39.8)$ & $60(17.5)$ \\
Vomiting & $59(29.4)$ & $43(12.5)$ \\
Splenomegaly & $51(25.4)$ & $40(11.7)$ \\
Hepatomegaly & $46(22.9)$ & $34(9.9)$ \\
Headache & $81(40.3)$ & $33(9.6)$ \\
Diarrhea & $27(13.4)$ & $19(5.5)$ \\
Rigors & $54(26.9)$ & $16(4.7)$ \\
Nausea & $34(16.9)$ & $16(4.7)$ \\
Abdominal pain & $52(25.9)$ & $13(3.8)$ \\
Dizziness & $33(16.4)$ & $8(2.3)$ \\
Sleep disorder & $33(16.4)$ & $3(0.9)$ \\
Fatigue & $62(30.8)$ &
\end{tabular}

Four-dose regimen: studies 003, 008, 009, 010, 011, and 025 .

Six-dose regimen: studies 025,026 , and 2403 .

Adverse events are listed according to decreasing frequency in the coartemether group.

$n$, number of patients with adverse events; $N$, total number of patients.

rates of individual AEs. Among the 14 most common AEs, 9 showed at least a 2-fold lower incidence with the six-dose than the four-dose regimen (Table 7). With regard to AEs of special interest, fewer patients in the six-dose than the four-dose group showed AEs related to the central nervous system. Of these, headache (six-dose: $9.9 \%$; four-dose: $40.3 \%$ ) and dizziness (six-dose: $3.8 \%$; four-dose: $16.4 \%$ ) were by far the most frequent ones. Other events included clonus in $3.8 \%$ of patients taking the six-dose regimen and $1.0 \%$ of those treated with the four-dose regimen, hyperreflexia (six-dose: $1.7 \%$; four-dose: $0.5 \%$ ), convulsions (six-dose: $0.3 \%$; four-dose: $1.0 \%$ ), and nystagmus, ataxia, and coordination abnormal, all of which occurred in $1.0-1.5 \%$ of patients treated with the four-dose regimen, but were not reported with the six-dose regimen. Decreased hearing (hypoacusis) was reported in $3 / 201(1.5 \%)$ of patients taking the four-dose regimen, but was not reported in patients taking the six-dose regimen. All cases of hypoacusis were of mild severity, and none were considered to be drug-related.

AEs suspected to be drug-related are shown in Table 8 . Only a rather small proportion of patients who experienced AEs had events that were suspected to be drug-related. Such AEs were overall higher with the coartemether six-dose regimen than the four-dose regimen.

Clinical laboratory parameters. The pattern of changes seen in clinical laboratory parameters was consistent with acute malaria and its resolution, with no striking differences between the four- and six-dose regimen of coartemether. With both regimens, hemoglobin levels and hematocrit decreased from baseline to day 3 and subsequently increased. In the six-dose group, a lower decrease to day 3 and a greater increase to day 28 was observed than in the four-dose group. Neither the six-dose regimen nor the four-dose regimen of coartemether seemed to have any clinically relevant effect on renal function, as assessed by serum creatinine levels. Paucity of data prevented comparisons with regard to other laboratory parameters. 
TABLE 8

Adverse events (after baseline but before recurrence of malaria) in at least $2 \%$ of patients receiving coartemether, suspected by the investigator to be drug-related (safety population)

\begin{tabular}{|c|c|c|}
\hline \multirow[b]{3}{*}{ Adverse event } & \multicolumn{2}{|c|}{$n(\%)$ patients } \\
\hline & \multicolumn{2}{|c|}{ coartemether } \\
\hline & $\begin{array}{l}\text { 4-dose regimen } \\
\quad(N=201)\end{array}$ & $\begin{array}{l}\text { 6-dose regimen } \\
\quad(N=343)\end{array}$ \\
\hline Total & $28(13.9)$ & $85(24.8)$ \\
\hline Anemia & $3(1.5)$ & $15(4.4)$ \\
\hline Clonus & $1(0.5)$ & $13(3.8)$ \\
\hline Vomiting & $3(1.5)$ & $13(3.8)$ \\
\hline Eosinophilia & 0 & $12(3.5)$ \\
\hline Diarrhea & $2(1.0)$ & $11(3.2)$ \\
\hline Rash & $1(0.5)$ & $9(2.6)$ \\
\hline Anorexia & $8(4.0)$ & $4(1.2)$ \\
\hline Sleep disorder & $4(2.0)$ & $1(0.3)$ \\
\hline Pyrexia & $4(2.0)$ & 0 \\
\hline Rigors & $4(2.0)$ & 0 \\
\hline
\end{tabular}

Electrocardiography. For both coartemether treatment regimens, the incidence in QTc changes within specific ranges were overall comparable for the six-dose and four-dose regimens (Table 9). Specifically, the proportion of patients who had clinically non-significant QTc changes of less than $30 \mathrm{~ms}$ from baseline was greater for the six-dose than the four-dose regimen. On the other hand, the four-dose regimen had greater proportions of patients with QTc increases of 30-60 or more than $60 \mathrm{~ms}$ than the six-dose regimen. This was generally consistent when QTc was calculated using either Bazett or Fridericia formulae. Given the low number of patients with ECG assessments in the four-dose group, differences between treatment groups should be interpreted with caution.

\section{DISCUSSION}

The present pooled analysis was performed to confirm that the six-dose regimen of coartemether is more efficacious than the four-dose regimen in children while remaining equally safe and well tolerated.

TABLE 9

Summary of QTc increases from baseline* (safety population)

\begin{tabular}{|c|c|c|}
\hline & \multicolumn{2}{|c|}{$n(\%)$ patients } \\
\hline & \multicolumn{2}{|c|}{ coartemether } \\
\hline & 4-dose regimen & 6-dose regimen \\
\hline Bazett's formula & $N=24$ & $N=291$ \\
\hline$<30 \mathrm{~ms} \dagger$ & $15(62.5)$ & $230(79.0)$ \\
\hline $30-60 \mathrm{~ms}$ & $8(33.3)$ & $49(16.8)$ \\
\hline$>60 \mathrm{~ms}$ & $1(4.2)$ & $12(4.1)$ \\
\hline Fridericia's formula & $N=17$ & $N=291$ \\
\hline$<30 \mathrm{~ms} \dagger$ & $7(41.2)$ & $193(66.3)$ \\
\hline $30-60 \mathrm{~ms}$ & $8(47.1)$ & $81(27.8)$ \\
\hline$>60 \mathrm{~ms}$ & $2(11.8)$ & $17(5.8)$ \\
\hline $\begin{array}{l}\text { Four-dose regimen: studi } \\
\text { Six-dose regimen: studie } \\
* \text { The highest increase re } \\
\dagger \text { Including decreases in } \\
N, \text { total number of patie } \\
\text { ralue. }\end{array}$ & $\begin{array}{l}010,011 \text {, and } \\
403 \text {. } \\
\text { me during the } \\
\text {, number of pa }\end{array}$ & table \\
\hline
\end{tabular}

The main outcomes of this pooled analysis of individual patient data from eight studies in children with acute, uncomplicated $P$. falciparum malaria, matched for demographic and baseline disease characteristics and individual coartemether doses, can be summarized as follows: 1) the parasitological cure rate at 28 days is clinically and statistically significantly higher with the six-dose than the four-dose regimen of coartemether; 2) the six-dose regimen is associated with a more rapid clearance of parasites and fever and a faster and more sustained reduction in gametocyte carriage; 3 ) patients in the six-dose group show a markedly lower discontinuation rate because of insufficient efficacy than patients on the four-dose regimen; 4) the six-dose regimen is equally well tolerated as the four-dose regimen in terms of AEs, SAEs, and laboratory parameters; 5) ECG changes (in terms of QTc prolongations) are infrequent and comparable between the two coartemether dose regimens.

Efficacy. This analysis showed that the six-dose regimen of coartemether is highly efficacious in the treatment of children with a body weight as low as $5 \mathrm{~kg}$, including those who are likely to be non-immune. Analyses based on the ITT and evaluable populations yielded PCR-corrected day 28 cure rates of $93 \%$ and $96 \%$, respectively. If compared with the four-dose regimen, cure rates were significantly higher with the six-dose regimen, irrespective of analysis population, whereas the results in the ITT population might have been slightly biased to the disadvantage of the four-dose regimen because of a higher proportion of patients being lost to follow-up, which were counted as treatment failures. Although PCT and FCT were shorter with the six-dose regimen, the proportion of patients with parasite clearance within 48 hours was high for both coartemether regimens. Given the fact that the two regimens are identical for the first 36 hours, this was not surprising.

Our findings confirmed the results from Falade and others ${ }^{14}$ who showed that the coartemether six-dose treatment of children with falciparum malaria is highly efficacious in endemic countries of Africa, using a non-comparative study design. Moreover, results from our analysis corroborate findings of a study in Thai adults that compared the six-dose and four-dose regimens directly ${ }^{10}$ and of a recently performed pooled analysis of data from adolescents and adults participating in randomized clinical trials (E. A. Mueller and others, unpublished data).

With regard to reduction of gametocyte carriage, in this analysis, the six-dose regimen acted faster and significantly fewer patients showed circulating gametocytes at day 28 than the four-dose regimen. Thus, the six-dose regimen may have a favorable impact on malaria transmission. Decreasing the transmission of any drug-resistant parasites that might have been selected is of major public health interest. ${ }^{5,21}$

Tolerability and safety. The safety profile of the six-dose regimen was not worse than that of the four-dose regimen. In fact, the six-dose regimen was well tolerated in the treatment of children down to $5-\mathrm{kg}$ body weight. The observation of lower AE rates with the six-dose regimen is most likely a reflection of the fact that most of the observed AEs were related to malaria symptoms, and the better efficacy associated with the six-dose regimen allowed a more rapid resolution of malaria and thus prevented worsening of symptoms more effectively than the four-dose regimen. This is supported by the fact that most AEs were reported during the 
first 3 days of treatment. Moreover, the six-dose regimen group was composed of more very small children with a limited ability to communicate subjective symptoms.

Suspected drug-related AEs were overall more frequent with the six-dose regimen of coartemether, which may look surprising as a lower proportion of AEs of any cause was observed with that regimen. However, this finding has to be interpreted with caution because drug relationship, according to the investigator's judgment, might have been assessed differently across studies. In addition, Study 2403, which provided most of the data on the six-dose regimen, followed an open, non-comparative design, in which the investigators knew they were prescribing a higher dose of coartemether than would previously have been used in the patient population represented in the study. This may have increased the index of suspicion. In contrast, almost one third of the patients in the pooled population for the four-dose regimen participated in double-blind comparative studies.

There has been some concern about the possible risk of neurotoxicity with artemisinin derivatives in animals and hearing decreases with coartemether in humans. ${ }^{22,23}$ In our pooled analysis in children, the six-dose regimen was not associated with a worse neurologic safety profile than the fourdose regimen. Headache and dizziness were by far the most frequent neurologic AEs and were almost certainly symptoms of malaria. Decreased hearing was not reported in patients taking the six-dose regimen, thereby confirming results of a recent study that indicated that coartemether has no influence on auditory function. ${ }^{24}$

SAEs were infrequent, and only one (severe urticaria) reported with the six-dose regimen was suspected to be drugrelated. One patient died of causes unrelated to study treatment.

In terms of clinical laboratory parameters, the information was limited, but findings were consistent with acute malaria and its resolution after treatment, without major differences between coartemether treatment regimens. In the six-dose group, a lower decrease to day 3 in hemoglobin levels and hematocrit and a greater increase to day 28 was observed than in the four-dose group, most likely because of the better therapeutic effect with the six-dose regimen.

Lumefantrine has some chemical similarities with halofantrine, a drug known to cause QTc interval prolongation. Consequently, QTc values were evaluated in this pooled analysis. Although the number of patients with ECG assessments in the four-dose group was small, the six-dose regimen of coartemether did not seem to be associated with a greater frequency of QTc prolongation than the low rate observed with the four-dose regimen. Significant prolongation of the QTc interval has been observed in young African children with malaria (at day 4 versus baseline) in patients being treated with coartemether, chloroquine, or sulfadoxine/pyrimethamine (SP). The QTc prolongation seemed to be independent of the treatment given but showed a weak correlation to both baseline parasitemia and temperature. ${ }^{25}$ In view of this, and the electrocardiographic safety of coartemether shown before in healthy volunteers and malaria patients, ${ }^{26,27}$ it seems unlikely that the administration of the six-dose regimen of coartemether represents an increased cardiac risk in the patient population studied.

Limitations. There was an imbalance between coartemether treatment groups with regard to the proportion of patients from double-blind studies compared with open-label studies. This might have influenced the assessment of drugrelationship of AEs. There were too few patients treated with the four-dose regimen in the 5 - to $<10$-kg body weight group to allow meaningful comparisons with the six-dose regimen for that particular body weight range. Moreover, Study 2403 provided the vast majority of patients on the six-dose regimen. Hence, the patient sample providing data for that regimen might have been not fully representative of patients with falciparum malaria from other geographical regions.

Conclusion. Based on this pooled analysis, the six-dose administration schedule of coartemether is more efficacious than the four-dose regimen in children with uncomplicated $P$. falciparum malaria while remaining equally well tolerated. The six-dose regimen is associated with high parasitological cure rates even in non-immune infants of body weight as low as $5 \mathrm{~kg}$. This pooled analysis supports the harmonized use of the six-dose regimen of coartemether in children as single global dosing regimen.

Received December 21, 2005. Accepted for publication February 6, 2006.

Acknowledgments: Biometry and statistical analysis was carried out by DATAMAP GmbH, Freiburg, Germany. We are grateful to Mrs. Mailis Virtanen for her help and support in conducting this analysis.

Financial support: This pooled analysis was funded by Novartis Pharma AG, Basel, Switzerland.

Disclosure: Some of the authors are Novartis employees and have no conflicts of interest concerning the work reported in this paper.

Authors' addresses: Michael Makanga, European and Developing Countries Clinical Trials Partnership (EDCTP), PO Box 19070, Francie van Zijl Drive, Parow Valley 7500, Cape Town, Tygerberg 7505, South Africa. Zul Premji, Department of Parasitology and Entomology, Muhimbili Medical Centre, PO Box 65001, Dar es Salaam, Tanzania. Catherine Falade, Department of Pharmacology and Therapeutics, College of Medicine, University of Ibadan, Ibadan, Nigeria. Juntra Karbwang, WHO, Avenue Appia 20, CH-1211 Geneva 27, Switzerland. Edgar A. Mueller, Institute for Clinical Pharmacology, Public Health Research Association Saxony, Medical Faculty, Technical University, Fiedlerstrasse 27, D-01307 Dresden, Germany, Email: Edgar.Mueller@mailbox.tu-dresden.de. Kim Andriano, Novartis Pharmaceuticals Corporation, One Health Plaza, East Hanover, NJ 07936-1080. Philip Hunt, Novartis Horsham Research Centre, Wimblehurst Road, Horsham, RH12 5AB, UK. Patricia Ibarra de Palacios, Novartis Pharma AG, Clinical Research, Lichtstrasse 35, CH-4056 Basel, Switzerland.

Reprint requests: Edgar A. Mueller, MD, Institute for Clinical Pharmacology, Public Health Research Association Saxony, Medical Faculty, Technical University, Fiedlerstrasse 27, D-01307 Dresden, Germany. E-mail: Edgar.Mueller@mailbox.tu-dresden.de.

\section{REFERENCES}

1. Breman JG, Egan A, Keusch GT, 2001. The intolerable burden of malaria: a new look at the numbers. Am J Trop Med Hyg 64 (Suppl): 4i-7i.

2. Snow RW, Guerra CA, Noor AM, Myint HY, Hay SI, 2005. The global distribution of clinical episodes of Plasmodium falciparum malaria. Nature 434: 214-217.

3. Greenwood BM, Bojang K, Whitty CJ, Targett GA, 2005. Malaria. Lancet 365: 1487-1498.

4. Roll Back Malaria/World Health Organization, 2004. Position of WHO's Roll Back Malaria Department on malaria treatment policy. Available at: http://www.who.int/malaria/docs/ who_apt_position.htm. Accessed 24 November 2005.

5. White NJ, 2004. Anti-malarial drug resistance. J Clin Invest 113: 1084-1092. 
6. Muheki C, McIntyre D, Barnes KI, 2004. Artemisinin-based combination therapy reduces expenditure on malaria treatment in KwaZulu Natal, South Africa. Trop Med Int Health 9: 959-966.

7. Malenga G, Palmer A, Staedke S, Kazadi W, Mutabingwa T, Ansah E, Barnes KI, Whitty CJ, 2005. Anti-malarial treatment with artemisinin combination therapy in Africa. BMJ 331: 706707.

8. Ashley EA, White NJ, 2005. Artemisinin-based combinations. Curr Opin Infect Dis 18: 531-536.

9. Kshirsagar NA, Gogtay NJ, Moorthy NS, Garg MR, Dalvi SS, Chogle AR, Sorabjee JS, Marathe SN, Tilve GH, Bhatt AD, Sane SP, Mull R, Gathmann I, 2000. A randomized, doubleblind, parallel-group, comparative safety, and efficacy trial of oral coartemether versus oral chloroquine in the treatment of acute uncomplicated Plasmodium falciparum malaria in adults in India. Am J Trop Med Hyg 62: 402-408.

10. Van Vugt M, Wilairatana P, Gemperli B, Gathmann I, Phaipun L, Brockman A, Luxemburger C, White NJ, Nosten F, Looareesuwan S, 1999. Efficacy of six doses of artemetherlumefantrine (benflumetol) in multidrug-resistant Plasmodium falciparum malaria. Am J Trop Med Hyg 60: 936-942.

11. Van Vugt M, Looareesuwan S, Wilairatana P, McGready R, Villegas L, Gathmann I, Mull R, Brockman A, White NJ, Nosten F, 2000. Artemether-lumefantrine for the treatment of multidrug-resistant falciparum malaria. Trans $R$ Soc Trop Med Hyg 94: $545-548$.

12. Lefevre G, Looareesuwan S, Treeprasertsuk S, Krudsood S, Silachamroon U, Gathmann I, Mull R, Bakshi R, 2001. A clinical and pharmacokinetic trial of six doses of artemetherlumefantrine for multidrug-resistant Plasmodium falciparum malaria in Thailand. Am J Trop Med Hyg 64: 247-256.

13. Piola P, Fogg C, Bajunirwe F, Biraro S, Grandesso F, Ruzagira E, Babigumira J, Kigozi I, Kiguli J, Kyomuhendo J, Ferradini L, Taylor W, Checchi F, Guthmann JP, 2005. Supervised versus unsupervised intake of six-dose artemether-lumefantrine for treatment of acute, uncomplicated Plasmodium falciparum malaria in Mbarara, Uganda: a randomised trial. Lancet 365: 1467-1473.

14. Falade C, Makanga M, Premji Z, Ortmann CE, Stockmeyer M, de Palacios PI, 2005. Efficacy and safety of artemetherlumefantrine $\left(\right.$ Coartem $\left.^{\circledR}\right)$ tablets (six-dose regimen) in African infants and children with acute, uncomplicated falciparum malaria. Trans $R$ Soc Trop Med Hyg 99: 459-467.

15. Fogg C, Bajunirwe F, Piola P, Biraro S, Checchi F, Kiguli J, Namiiro P, Musabe J, Kyomugisha A, Guthmann JP, 2004. Adherence to a six-dose regimen of artemether-lumefantrine for treatment of uncomplicated Plasmodium falciparum malaria in Uganda. Am J Trop Med Hyg 71: 525-530.

16. Von Seidlein L, Jaffar S, Pinder M, Haywood M, Snounou G, Gemperli B, Gathmann I, Royce C, Greenwood B, 1997. Treatment of African children with uncomplicated falciparum malaria with a new anti-malarial drug, CGP 56697. J Infect Dis 176: 1113-1116.

17. Von Seidlein L, Bojang K, Jones P, Jaffar S, Pinder M, Obaro S, Doherty T, Haywood M, Snounou G, Gemperli B, Gathmann I, Royce C, McAdam K, Greenwood B, 1998. A randomized controlled trial of artemether/benflumetol, a new anti-malarial and pyrimethamine/sulfadoxine in the treatment of uncomplicated falciparum malaria in African children. Am J Trop Med Hyg 58: 638-644.

18. Hatz C, Abdulla S, Mull R, Schellenberg D, Gathmann I, Kibatala P, Beck HP, Tanner M, Royce C, 1998. Efficacy and safety of CGP 56697 (artemether and benflumetol) compared with chloroquine to treat acute falciparum malaria in Tanzanian children aged 1-5 years. Trop Med Int Health 3: 498-504.

19. Van Vugt M, Brockman A, Gemperli B, Luxemburger C, Gathmann I, Royce C, Slight T, Looareesuwan S, White NJ, Nosten F, 1998. Randomized comparison of artemether-benflumetol and artesunate-mefloquine in treatment of multidrug-resistant falciparum malaria. Antimicrob Agents Chemother 42: 135139.

20. Taylor WRJ, White NJ, 2004. Anti-malarial drug toxicity. Drug Saf 27: 25-61.

21. Sutherland CJ, Ord R, Dunyo S, Jawara M, Drakeley CJ, Alexander N, Coleman R, Pinder M, Walraven G, Targett GA, 2005. Reduction of malaria transmission to anopheles mosquitoes with a six-dose regimen of coartemether. PLoS Med 2: e92.

22. Brewer TG, Peggins JO, Grate SJ, Petras JM, Levine BS, Weina PJ, Swearengen J, Heiffer MH, Schuster BG, 1994. Neurotoxicity in animals due to arteether and artemether. Trans $R$ Soc Trop Med Hyg 88 (Suppl 1): S33-S36.

23. Toovey S, Jamieson A, 2004. Audiometric changes associated with the treatment of uncomplicated falciparum malaria with coartemether. Trans $R$ Soc Trop Med Hyg 98: 261-267.

24. Hutagalung R, Htoo $\mathrm{H}$, Nwee $\mathrm{P}$, Arunkmomkiri J, Zwang J, Carrara V, Ashley E, Singhasivanon P, White N, Nosten F, 2006. A case control auditory evaluation of patients treated with artemether-lumefantrine. Am J Trop Med Hyg 74: 211214.

25. Von Seidlein L, Jaffar S, Greenwood B, 1997. Prolongation of the QTc interval in African children treated for falciparum malaria. Am J Trop Med Hyg 56: 494-497.

26. Bindschedler M, Lefevre G, Ezzet F, Schaeffer N, Meyer I, Thomsen MS, 2000. Cardiac effects of coartemether (artemether/lumefantrine) and mefloquine given alone or in combination to healthy volunteers. Eur J Clin Pharmacol 56: 375381.

27. Van Vugt M, Ezzet F, Nosten F, Gathmann I, Wilairatana P, Looareesuwan S, White NJ, 1999. No evidence of cardiotoxicity during anti-malarial treatment with artemether-lumefantrine. Am J Trop Med Hyg 61: 964-967. 